\title{
Implementation of a checklist to increase adherence to evidence-based practices in a single pediatric intensive care unit
}

\author{
Pablo Eulmesekian, M.D. ${ }^{a}$, AugustoPérez,M.D. ${ }^{a}$, SilviaDíaz,M.D. ${ }^{a}$ and MateoFerrero, M.D. ${ }^{a}$
}

\begin{abstract}
Introduction. The use of checklists to increase adherence to evidence-based practices is not yet widespread in pediatric intensive care units. The objective of this study was to achieve $90 \%$ compliance with studied practices using an ad hoc checklist.
\end{abstract}

Population and methods. Time series quasiexperimental study conducted in ventilated children hospitalized in the pediatric intensive care unit. Studied practices included sedation breaks, plateau pressure $\leq 30 \mathrm{~cm} \mathrm{H}_{2} \mathrm{O}$, fraction of inspired oxygen $\leq 60 \%$, maintenance of headboard at $>30^{\circ}$, chlorhexidine mouthwash, weekly ventilator circuit changes, preference for enteral feeding, reduction in the threshold for blood transfusions (hemoglobin: $7 \mathrm{~g} / \mathrm{dL}$ ), daily consideration of spontaneous breathing trials and central venous catheter removal. The checklist was used during ward rounds by the staff physicians in charge of the pediatric intensive care unit as part of an intervention to increase adherence and as a tracking tool. Each form completed on a daily basis was considered an observation. Observations were classified as defective in the case of non-compliance with one or more items. Adherence (the rate of nondefective units of observation) is summarized in the control chart.

Results. The study period lasted 420 days. A total of 732 patients were hospitalized; 218 underwent mechanical ventilation; 1201 observations were made, and 1191 were included in the study. The control chart with a 14-month time horizon showed increased adherence, a special cause variation pattern in the last 3 months of the study period, and $>90 \%$ compliance over the last 2 months.

a. Department of Intensive Care of Hospital Italiano de Buenos Aires, Autonomous City of Buenos Aires.

E-mail address:

Pablo Eulmesekian, M.D.: pablo.eulmesekian@ hospitalitaliano.org.ar

Funding:

None.

Conflict of interest:

None.

Received: 12-5-2016

Accepted: 4-17-2017

\section{INTRODUCTION}

The Institute of Medicine (USA) defines "health care quality" as the degree to which health services for individuals and populations increase the likelihood of desired health outcomes that are consistent with current professional knowledge, therefore establishing a relationship between this concept and adherence to evidence-based practices. ${ }^{1}$ Yet compliance with practices that enhance intensive care unit outcomes is inconsistent. For different reasons, some patients are not benefiting from this intervention. ${ }^{2-4}$

Pronovost studied the impact of non-compliance with treatments that improved survival among adults admitted to the intensive care unit and estimated that almost 170000 lives could be saved each year if the studied evidence-based practices were fulfilled. ${ }^{5}$

Checklists help to prevent omission errors and are a useful tool to complete a task as simple as shopping or as complex as flying a plane. ${ }^{6}$ Their use has extended to different areas of medicine, for example, to diagnose brain death, ${ }^{7}$ increase treatment adherence after a myocardial infarction or a stroke ${ }^{8}$ or help to reduce intraoperative mortality. ${ }^{9}$

Although the use of checklists in intensive care settings has been disseminated, it is not widely spread. In the case of adults, checklists are used to improve communication, the fulfillment of goals, ${ }^{10}$ and as a tool to increase compliance with evidencebased practices. ${ }^{11-15}$ There are fewer references on their use in pediatric intensive care settings. Checklists have been used to improve the conveyance 
of health care goals ${ }^{16}$ and optimize the compliance with patient safety practices. ${ }^{17}$ In our setting, their use has not been reported.

The primary objective of this study was to achieve $90 \%$ adherence to evidence-based practices in ventilated children hospitalized in the pediatric intensive care unit (PICU) of Hospital Italiano de Buenos Aires (HIBA) using an ad hoc checklist. Secondary objectives were to evaluate the evolution of such compliance during the study period and to identify those practices with the highest and lowest percentage of adherence.

\section{POPULATION AND METHODS \\ Setting}

The PICU of HIBA is a multidisciplinary 20-bed unit, with an average of 750 annual admissions, and a rate of use of assisted mechanical ventilation (AMV) of $40 \%$. It is part of a university general hospital accredited by the Joint Commission International.

Ward rounds are a key activity of the unit. These are held every morning by multidisciplinary health care providers. Four staff physicians rotate weekly to run the PICU and lead ward rounds. They make therapeutic and diagnostic decisions and define health care objectives during these rounds.

\section{Design, population, and study period}

This was a time-series quasi-experimental study. The study time horizon was 12 months plus the pilot test period, and it was conducted between August $12^{\text {th }}, 2013$ and October $5^{\text {th }}$, 2014. The study population was made up of patients with AMV hospitalized in the PICU. Children older than 18 years old and those with a tracheostomy and not receiving sedatives by continuous intravenous infusion were excluded.

\section{Studied practices}

The evidence-based practices included in the checklist were:

- Daily interruption of sedation. ${ }^{18,19}$

- Lung-protective strategies (plateau pressure $\leq 30 \mathrm{~cm} \mathrm{H} \mathrm{H}_{2} \mathrm{O}$ and fraction of inspired oxygen $-\mathrm{FiO}-\leq 60){ }^{20}$

- Measures to prevent ventilator-associated pneumonia: elevation of the bed headboard at more than $30^{\circ}$, chlorhexidine $0.12 \%$ mouthwash every 8 hours, and ventilator circuit changes every 7 days as per the manufacturer's recommendations. ${ }^{21-23}$

- Daily consideration of weaning from the ventilator, determining whether the patient is ready to perform a spontaneous breathing trial. $^{24,25}$

- Daily consideration of removing the central venous catheter. ${ }^{14,15,26}$

- Preference for enteral feeding. 27,28

- Reduced threshold for red blood cell transfusions, with a hemoglobin concentration of $7 \mathrm{~g} / \mathrm{dL} \cdot{ }^{29,30}$

- Formulation of clear goals during ward rounds. ${ }^{10}$

- Medication reconciliation..$^{31,32}$

\section{Stages of the study \\ Design of the checklist}

A form was designed to include the patient's demographic data, studied practices, and a text field to record the reasons for non-fulfillment. The 4 staff physicians, responsible for the PICU and the implementation of the checklist, reviewed the draft form.

\section{Pilot test and periodic assessments}

The pilot test of the checklist was performed between August 12 ${ }^{\text {th }}, 2013$ and September 21 ${ }^{\text {st }}$, 2013. Problems were identified in the design of the form and modifications were introduced (Annex). The assessment was repeated every two months before the final assessment. During each assessment, the percentage of adherence was verified and items with the lowest percentage of fulfillment were identified. This information was submitted by the main author to the physicians using the checklist to encourage compliance with its implementation.

\section{Use of the checklist}

The checklist was used during ward rounds among all ventilated patients. After the explanation by the medical resident and once daily goals were established, the staff physician in charge of the PICU during that week would read each item out loud and record compliance or non-compliance with such item on the checklist. If any of the items had not been fulfilled, the staff physician would indicate it based on his/her prerogative.

\section{Unfulfilled items}

To define non-compliance with any of the practices, items described as unfulfilled were identified and checked to determine if there was a reason for such non-compliance. If there was an explanation, the item was considered fulfilled; 
e.g., if a patient was in the prone position, the person completing the form would write next to "Headboard at more than $30^{\circ}$ " that it was not possible to adhere to this practice because of the patient's position. On the contrary, if the person completing the form omitted to provide an explanation, the item was considered unfulfilled. The presence of at least one unfulfilled item rendered the observation defective. Reasons for non-compliance were not standardized.

\section{Definition of outcome measures}

- Unit of observation: every form filled in during ward rounds in each ventilated patient.

- Items included in the unit of observation: every practice included in the checklist.

- Fulfilled items: items effectively implemented or those for which non-compliance is duly justified.

- Unfulfilled items: items indicated as not implemented for no reason or without a duly justified reason.

- Defective unit of observation: that with at least one unfulfilled item.

- Non-defective unit of observation: that in which all items were classified as fulfilled.

- Rate of defective units: ratio between the number of defective units and the number of observations.

- Rate of non-defective units (indicator of adherence): ratio between the number of non-defective units and the number of observations.

\section{Indicators of outcome}

Mortality, rate of AMV and central venous catheter use, rate of ventilator-associated pneumonia and central venous catheter-related bacteremia, percentage of prescription errors, and episodes of drops were recorded simultaneously.

\section{Statistical analysis}

Summary measures included mean and standard deviation (SD) for outcome measures with a normal distribution, interquartile range for those with an abnormal distribution, and rates for categorical outcome measures.

Control charts were used to analyze the percentage of adherence to studied practices based on time. They allow to establish a difference among common cause variation, random variation, and special cause variation. Point distribution in the chart accounts for such difference. A consistent pattern of 1 point outside the control limits, 6 points in a row -all increasing or all decreasing- or 8 points in a row on the same side of the center line define special cause variation. If the control chart shows special cause variation, it is possible to deduce that there is a very low probability that changes have occurred by chance. If the control limits of the control chart are set at $\pm 3 \mathrm{SD}$, type I error is 0.0027 for each measurement and $\left(1-0.9973^{\text {n measurements }}\right)$ for the whole chart. 33,34

In this study, a type P chart was used with biweekly measurements and a 14-month time horizon. Control limits were set at \pm 3 SD. The statistical analysis was performed with the Stata 9 software (Statacorp, Texas).

\section{Ethical aspects}

Collected data were managed in agreement with the Protection of Personal Information Act. The protocol was approved by the Research Protocol Assessment Committee of Hospital Italiano de Buenos Aires, which also authorized the waiver of informed consent (Protocol no. 1999, dated January $17^{\text {th }}, 2013$ ).

\section{RESULTS}

The study period lasted 420 days, between August $12^{\text {th }}, 2013$ and October $5^{\text {th }}, 2014$. In this period, 732 patients were hospitalized, 218 received AMV, 1201 observations were made, and 10 of these were excluded due to missing items (sedation breaks: 3 ; heater on: 2; enteral feeding: 2; oral hygiene: 1 ; RBC transfusion: 1 ; and $\mathrm{FiO}_{2}<60 \%$ : 1). A total of 1191 observations were included in this study. The characteristics of the population are summarized in Table 1. During the study, 6 bimonthly assessments were carried out. The first assessment was done after finishing the pilot test. After this initial assessment, the checklist items were dichotomized.

Out of the 1191 observations included in the study, $183(15.3 \%)$ were defective, i.e., they indicated non-adherence to at least one of the studied practices. Among these, 163 (89.1\%) showed non-adherence to 1 practice; $18(9.8 \%)$, to 2 practices; and $2(1.1 \%)$, to 3 practices.

The evolution of the percentage of adherence over time or the rate of non-defective units of observation are summarized in Figure 1. The figure shows a mean of $85 \%$ adherence and a common cause variation pattern involving up to the last 8 points in the chart. These are above the center line and result in a special cause variation pattern corresponding to the last 3 months 
of the study period. The last 6 measurements show $>90 \%$ adherence. Figure 2 shows overall adherence and the percentage of defective units of observation on a bimonthly basis.

Practices with the lowest percentage of adherence were interruption of sedation and headboard position, with an average of $5.2 \%$ and $4.6 \%$ non-compliance, respectively. Practices with the highest percentage of adherence were plateau pressure $<30 \mathrm{~cm} \mathrm{H}_{2} \mathrm{O}$, ventilator circuit change within 7 days of use, and oral hygiene, with an average of more than $99 \%$ compliance, and $\mathrm{FiO}_{2}<60 \%$, with $98.2 \%$ compliance. Table 2 shows the bimonthly development of outcome indicators.

TABLE 1. Characteristics of the population under study

\begin{tabular}{lc}
\hline Outcome measure & \\
\hline Observations, $\mathrm{n}$ & 1191 \\
Ventilated patients, $\mathrm{n}$ & 218 \\
Age in years, median (p 25-p 75) & $1.2(0.4-3.3)$ \\
Sex, male, \% & 52.7 \\
Weight in kg, median (P 25-P 75) & $8.8(5.5-13)$ \\
PIM2, \% ( \pm SD) & $7.8( \pm 13.73)$ \\
Underlying disease, \% & 89.6 \\
Post-surgical AMV, \% & 42.9 \\
Days of AMV, median (p 25-p 75) & $4(2-8)$ \\
Mortality, \% & 16.5 \\
\hline
\end{tabular}

PIM2: Pediatric Index of Mortality 2.

AMV: assisted mechanical ventilation.

SD: standard deviation.

\section{DISCUSSION}

The use of a checklist among ventilated children hospitalized in the PICU of HIBA increased the adherence to the studied evidencebased practices and achieved more than $90 \%$ compliance over the last 2 months of the study period. Given that the control chart showed the occurrence of a special cause variation and that the checklist was an intervention aimed at modifying process variations, it is possible to infer that such increase was associated with its use.

With the use of the checklist, two goals were pursued. The primary objective was to establish an intervention oriented at improving adherence to the studied practices; the secondary objective was to use it as a tracking tool necessary to define outcome measures and indicators. Only 4 staff physicians used the checklist because they were the ones who rotated weekly to be in charge of the PICU, were the heads of the ward rounds, and had the prerogative to indicate compliance with the studied practices. Restricting the checklist management to this small group was part of a strategy considered at the time of designing the study to reduce variability in its use and, together with bimonthly meetings, to encourage commitment to its fulfillment. Weiss demonstrated that using checklists only improved outcomes if staff members were committed to complete it and did not take it as bureaucracy. ${ }^{35}$

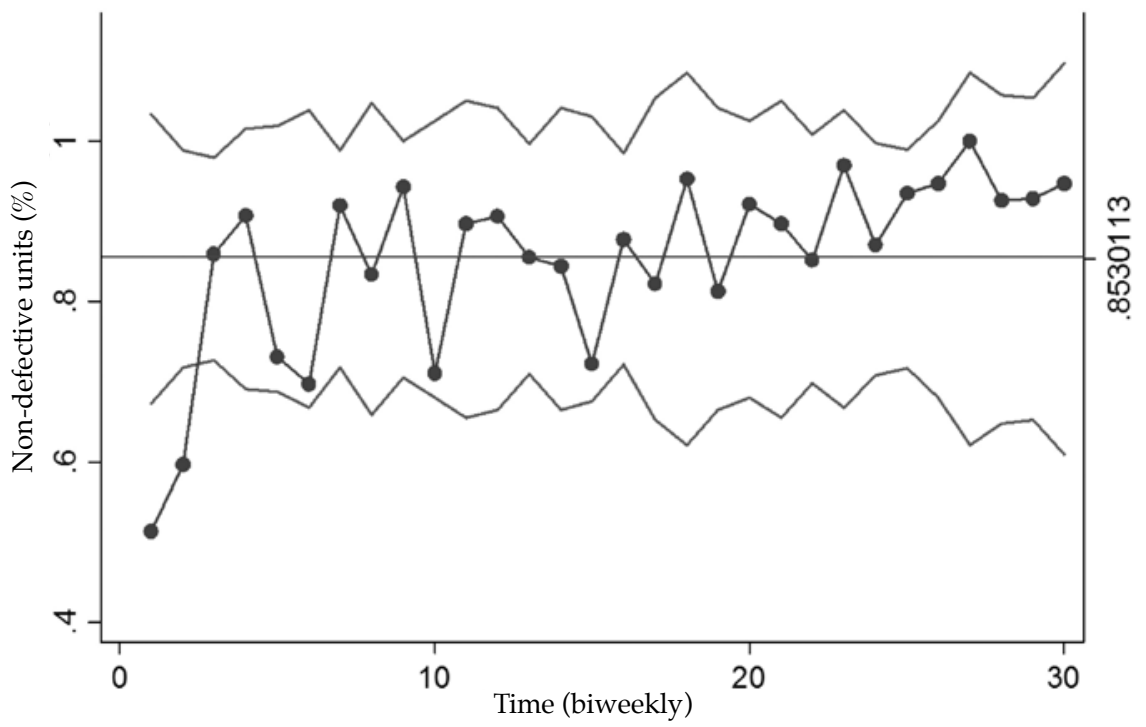

Horizontal line: mean adherence.

Upper and lower lines: control limits of the chart set at \pm 3 SD. 
The control chart showed common cause variation in the percentages of adherence during most of the study period, except on two occasions. The first time corresponded to the 2 baseline measurements made during the pilot test, which were below the lower control limit and showed approximately $50 \%$ adherence, i.e., the lowest values obtained during the study. Although these measurements corresponded to the pilot test of the checklist, they were included because they were significant. Although probably less accurate than subsequent measurements, these showed that studied patients had not benefited from the set of studied practices during half of the period while they were receiving AMV. This helped to describe the initial size of this problem. The second time a special cause variation was observed, it corresponded to the last 3 months of the study period. Over this 3-month period, 8 biweekly measurements were above the center line. ${ }^{33,34}$ This means that the variation pattern of the process had changed, adherence increased, and was not the result of a random factor.

The simultaneous development of outcome indicators showed that, over the study period, there was a reduction in the rate of ventilatorassociated pneumonia and central venous catheter-related bacteremia, and in the rate of central venous catheter use. Although it is not possible to state that it is associated with the use of the checklist, such development enables to infer that forms were completed in a committed manner and that it is not very likely that there has been a bias when completing them.

There are a few published studies on the use of checklists in relation to children hospitalized in the PICU. Tarrago et al. reported on the use of checklists to increase adherence to practices aimed at enhancing patient safety over 21 months. The checklist was used by a reduced group made up of 5 staff physicians during ward rounds; the authors found a greater adherence to studied

TABLE 2. Bimonthly development of outcome indicators

\begin{tabular}{|c|c|c|c|c|c|c|c|c|c|c|}
\hline \multirow[t]{2}{*}{ Indicator } & \multicolumn{3}{|c|}{ PRE } & \multicolumn{7}{|c|}{ POST } \\
\hline & $\begin{array}{c}\text { March } \\
\text { April } 2013\end{array}$ & $\begin{array}{c}\text { May } \\
\text { June } 2013\end{array}$ & $\begin{array}{c}\text { July } \\
\text { Aug. } 2013\end{array}$ & $\begin{array}{c}\text { Sep. } \\
\text { Oct. } 2013\end{array}$ & $\begin{array}{c}\text { Nov. } \\
\text { Dec. } 2013\end{array}$ & $\begin{array}{c}\text { Jan. } \\
\text { Feb. } 2014\end{array}$ & $\begin{array}{c}\text { March } \\
\text { April } 2014\end{array}$ & $\begin{array}{c}\text { May } \\
\text { June } 2014\end{array}$ & $\begin{array}{c}\text { July } \\
\text { Aug. } 2014\end{array}$ & $\begin{array}{c}\text { Sep. } \\
\text { Oct. } 2014\end{array}$ \\
\hline Mortality, \% & 5.7 & 3.3 & 4.1 & 4.3 & 4.5 & 5.7 & 4.6 & 6.3 & 5.4 & 5.2 \\
\hline AMV use, $\%$ & 49 & 37 & 29 & 32 & 32 & 43 & 40 & 31 & 39 & 35 \\
\hline VAP rate* & 4.9 & 2.9 & 3.9 & 3.3 & 0 & 0 & 7.2 & 0 & 0 & 0 \\
\hline CVC use, $\%$ & 66 & 66 & 67 & 72 & 61 & 62 & 61 & 59 & 52 & 53 \\
\hline CVCRB rate & 12.5 & 0 & 6.8 & 6 & 4.1 & 6.6 & 4.7 & 0 & 5.2 & 2.7 \\
\hline $\mathrm{PE}, \%$ & 1.4 & 3.1 & 1.5 & 1.2 & 1.4 & 1.8 & 1.5 & 2.2 & 1.5 & 1.7 \\
\hline Drops & 0 & 0 & 0 & 0 & 1 & 0 & 0 & 0 & 0 & 0 \\
\hline
\end{tabular}

AMV: assisted mechanical ventilation; VAP: ventilator-associated pneumonia; CVC: central venous catheter; CVCRB: central venous catheter-related bacteremia; PE: prescription errors.

* VAP rate: ventilator-associated pneumonia episodes per every 1000 days of assisted mechanical ventilation. + CVCRB rate: central venous catheter-related bacteremia episodes per every 1000 days of central venous catheter use. PRE: period before the intervention; POST: period after the intervention.

FIGURE 2. Bimonthly evolution of overall adherence percentages and defective units of observation

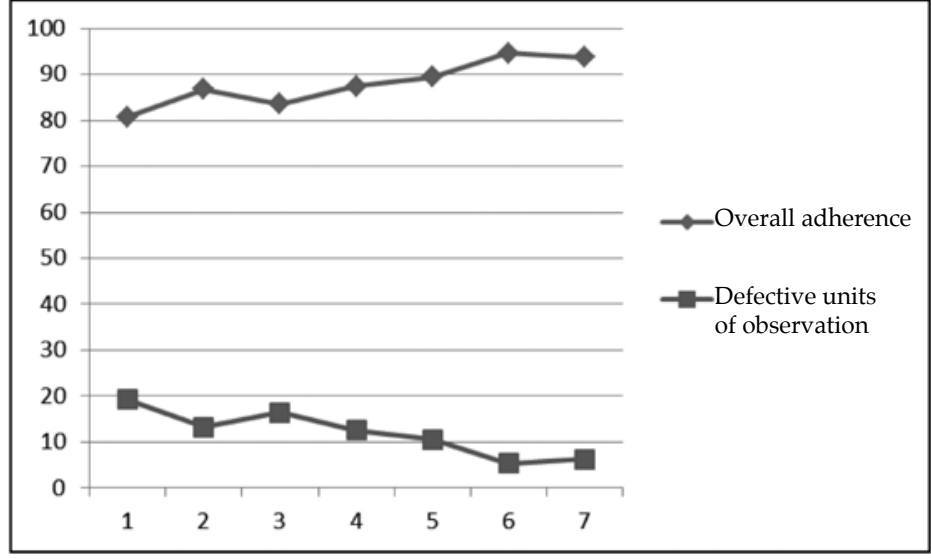


practices and a positive development of outcome indicators, such as the rate of use of catheters, arterial lines, urinary catheters, and intravenous antibiotics, and the number of lab tests done. These authors were concerned that the checklist was not completed as part of bureaucracy so they presented, before all the PICU staff, the times physicians had failed to complete the form and also the evolution of recorded indicators. They considered that such strategy (exposing faults and generating competition among peers) had a positive effect and resulted in the commitment of physicians to fill in the checklist. ${ }^{17}$ With different cultural characteristics though with the same objective, in this study physicians using the checklist received periodic, limited reports on its use.

This study poses limitations. On the one side, given that this study was conducted at a PICU, with specific characteristics in terms of organization and patient population, findings cannot be extrapolated. On the other side, there was not a control group, so it is not possible to ascertain that findings are exclusively associated with the use of the checklist. In addition, since the justifications for not adhering to a practice were not standardized, it may have resulted in variations in the reasons for not complying with the items included in the checklist. However, it was designed to be used by a reduced number of physicians, who shared ward rounds and had been working together for years, so as to reduce bias in relation to lack of standardization. The registration of inadequate justifications for non-compliance could have also been biased, which would lead to considering unfulfilled practices as fulfilled. This may have resulted in over-estimation of the percentage of adherence. Notwithstanding this, the positive development of certain indicators makes it possible to deduce that reported outcomes are reliable.

This study also has strengths. Most importantly, this is an easy to implement intervention, it does not involve excessive costs nor risks to the patients, and may potentially improve outcomes and the quality of health care provided.

\section{CONCLUSIONS}

The daily use of a checklist to increase adherence to evidence-based practices (sedation breaks, lung-protective ventilation strategies, ventilator-associated pneumonia prevention, preference for enteral feeding, reduction in the threshold for blood transfusions, daily consideration of spontaneous breathing trials and central venous catheter removal), improved adherence to these practices and achieved more than $90 \%$ compliance during the last 2 months of the study period.

\section{REFERENCES}

1. Institute Of Medicine. America's health in transition: protecting and improving quality. Washington DC: National Academy Press; 1994:3-5. [Accessed on: August $24^{\text {th }}$, 2015]. Available at: http: / / www.nap.edu/catalog/9147 / americas-health-in-transition-protecting-and-improvingquality.

2. HesdorfferDC, GhajarJ.Marked improvement in adherence to traumatic brain injury guidelines in United States trauma centers. J Trauma 2007;63(4):841-7.

3. Joynes E, Dalay S, Patel JM, et al. A complete audit cycle to assess adherence to a lung protective ventilation strategy. Indian J Crit Care Med 2014;18(11):746-9.

4. Gurses AP, Seidi KL, Vaidya V, et al. Systems ambiguity and guideline compliance: a qualitative study of how intensive care units follow evidence-based guidelines to reduce healthcare-associated infections. Qual Saf Health Care 2008;17(5):351-9.

5. Pronovost PJ, Rinke ML, Emery K, et al. Interventions to reduce mortality among patients treated in intensive care units. J Crit Care 2004;19(3):158-64.

6. Winters BD, Gurses AP, Lehmann $\mathrm{H}$, et al. Clinical review: Checklists - translating evidence into practice. Crit Care 2009;13(6):210.

7. Young GB, Frewen T, BarrHW, et al. Checklist for diagnosis of brain death. Can J Neurol Sci 1991;18(1):104.

8. Wolff AM, Taylor SA, McCabe JF. Using checklists and reminders in clinical pathways toimprove hospital inpatient care. Med J Aust 2004;181(8):428-31.

9. Haynes AB, Weiser TG, Berry WR, et al. A surgical safety checklist to reduce morbidity and mortality in a global population. N Engl J Med 2009;360(5):491-9.

10. Pronovost P, Berenholtz S, Dorman T, et al. Improving communication in the ICU using daily goals. J Crit Care 2003;18(2):71-5.

11. Berenholtz SM, Milanovich S, Faircloth A, et al. Improving care for the ventilated patient. It Comm J Qual Saf 2004;30(4):195-204.

12. Byrnes MC, Schuerer DJ, Schallom ME, etal.Implementation of a mandatory checklist of protocols and objectives improves compliance with a wide range of evidence based intensive care unit practices. Crit Care Med 2009;37(10):2775-81.

13. Dubose J, Teixeira PG, Inaba K, et al. Measurable outcomes of quality improvement using a daily quality rounds checklist: one year analysis in a trauma intensive care unit with sustained ventilator associated pneumonia reduction. J Trauma 2010;69(4):855-60.

14. Berenholtz SM, Pronovost PJ, Lipsett PA, et al. Eliminating catheter-related bloodstream infections in the intensive care unit. Crit Care Med 2004;32(10):2014-20.

15. Pronovost P, Needham D, Berenholtz S, et al. An intervention to decrease Catheter Related Bloodstream Infections in the ICU. N Engl J Med 2006;355(26):2725-32.

16. Agarwal S, Frankel L, Tourner S, et al. Improving communication in a pediatric intensive care unit using daily patient goal sheets. J Crit Care 2008;23(2):227-35.

17. Tarrago R, Nowak JE, Leonard CS, et al. Reductions in invasive device use and care costs after institution of a daily 
safety checklist in a pediatric critical care unit. Jt Comm J Qual Patient Saf 2014;40(6):270-8.

18. Gupta K, Gupta VK, Jayashree M, et al. Randomized controlled trial of interrupted versus continuous sedative infusions in ventilated children. Pediatr Crit Care Med 2012;13(2):131-5.

19. KressJP,Pohlman AS, $\mathrm{O}^{\prime}$ ConnorMF, etal.Daily interruption of sedative infusions in critically ill patients undergoing mechanical ventilation. N Engl J Med 2000;342(20):1471-7.

20. PetrucciN, DeFeoC. Lung protective ventilation strategy for the acute respiratory distress syndrome. Cochrane Database Syst Rev 2013;2:CD003844.

21. Curley MA, Schwalenstocker E, Deshpande JK, et al. Tailoring the Institute for Health Care Improvement 100,000 Lives Campaign to pediatric settings: the example of ventilator-associated pneumonia. Pediatr Clin North Am 2006;53(6):1231-51.

22. Bigham MT, Amato $R$, Bondurrant $\mathrm{P}$, et al. Ventilator associated pneumonia in the pediatric intensive care unit: characterizing the problem and implementing a sustainable solution. J Pediatr 2009;154(4):582-7.e2.

23. Fisher \& Paykel Healthcare. Evaqua Breathing Circuits. [Accessed on: October 16 $\left.{ }^{\text {th }}, 2014\right]$. Available at: https:/ / www.fphcare.co.nz/products/evaqua-circuits.24.

24. McConville JF, Kress JP. Weaning patients from the ventilator. N Engl J Med 2012; 367(23):2233-9.

25. Foronda FK, Troster EJ, Farias JA, et al. The impact of daily evaluation and spontaneous breathing test on the duration of pediatric mechanical ventilation: a randomized controlled trial. Crit Care Med 2011;39(11):2526-33.

26. Ahmed SS, McCaskey MS, Bringman S, et al. Catheter associated bloodstream infection in the pediatric intensive care unit: a multidisciplinary approach. Pediatr Crit Care
Med 2012;13(2):e69-72.

27. McClaveSA, MartindaleRG, VanekVW, etal.Guidelines for the provision and assessment of nutrition support therapy in the adult critically ill patient: Society of Critical Care Medicine and American Society for Parenteral and Enteral Nutrition. JPEN J Parenter Enteral Nutr 2009;33(3):277-316.

28. Mehta NM, Bechard LJ, Cahill N, et al. Nutritional practices and their relationship to clinical outcomes in critically ill children: an international multicenter cohort study. Crit Care Med 2012;40(7):2204-11.

29. Lacroix J, Hébert PC, Hutchinson JS, et al. Transfusion strategies for patients in pediatric intensive care units. $N$ Engl J Med 2007;356(16):1609-19.

30. Rouette J, Trottier H, Ducruet T, et al. Red blood cell transfusion threshold in postsurgical pediatric intensive care patients: a randomized clinical trial. Ann Surg 2010;251(3):421-7.

31. Manias E, Williams A, Liew D. Interventions to reduce medication errors in adult intensive care: a systematic review. Br J Clin Pharmacol 2012;4(3):411-23.

32. Pronovost P, Weast B, Schwarz M, et al. Medication reconciliation: a practical tool to reduce the risk of medication errors. J Crit Care 2003;18(4):201-5.

33. Benneyan JC, Lloyd RC, PlsekPE. Statistical process control as a tool for research and healthcare improvement. Qual Saf Health Care 2003;12(6):458-64.

34. Mohammed MA, Worhtington P, Woodall WH. Plotting basic control charts: tutorial notes for healthcare practitioners. Qual Saf Health Care 2008;17(2):137-45.

35. Weiss $\mathrm{CH}$, Moazed F, McEvoy CA, et al. Prompting physicians to address a daily checklist and process of care and clinical outcomes. Am J Respir Crit Care Med 2011;184(6):680-6. 
ANNEX

Checklist

\begin{tabular}{|l|l|}
\hline Date & \\
\hline Observation number & \\
\hline Patient's last name & \\
\hline
\end{tabular}

Read out loud and fill in during ward rounds among ventilated patients

(Except when they have a tracheostomy and are not receiving intravenous sedatives)

Received standard of care

\begin{tabular}{|c|c|c|c|}
\hline & Yes & No & Reason* \\
\hline Indication and performance of sedation breaks & 0 & 0 & \\
\hline Sedation break performed in the previous 24 hours & 0 & 0 & \\
\hline Plateau pressure $\leq 30 \mathrm{~cm} \mathrm{H}_{2} 0$ & 0 & 0 & \\
\hline $\mathrm{FiO}_{2} \leq 60 \%$ & 0 & 0 & \\
\hline
\end{tabular}

Heated humidifier on

Clean tubing (see change date)

Headboard at more than $30^{\circ}$

Indication of oral hygiene

Oral hygiene performed in the previous 24 hours

Evaluation of spontaneous breathing trial

Evaluation of CVC removal

Received enteral feeding

Standard transfusion (hematocrit $<21 \%$ )

Medication reconciliation

00

00

○ 0

00

00

○ 0

00

00

00

00

* If any of these practices was omitted, please explain the reason. 